\title{
Experience of Palliative Radiotherapy for Tumor-Associated Bleeding From Biliary Tract Cancer
}

\author{
Hideaki Kawabata, d, Misuzu Hitomi ${ }^{\text {a }}$, Yoshiki Yamamoto ${ }^{\mathrm{b}}$, Takashi Fujiic, \\ Naonori Inoue $^{a}$, Yukino Kawakatsu ${ }^{\text {a }}$, Yuji Okazaki ${ }^{a}$, Masatoshi Miyata ${ }^{a}$, \\ Shigehiro Motoi ${ }^{\mathrm{a}}$, Yoshihiro Shimizu ${ }^{\mathrm{b}}$
}

\begin{abstract}
We experienced two cases of palliative radiotherapy (RT) for tumorassociated bleeding from biliary tract cancer (BTC). Bleeding due to tumor invasion to the duodenum wall caused severe anemia that required a blood transfusion. Hemostatic RT with 30 Gy in $10-12$ fractions was attempted. Hemostasis was achieved in both patients, and they were able to spend their remaining life at home with no restrictions of their oral intake. In addition, no adverse events were seen in any of the two patients after undergoing palliative RT. Palliative RT for tumor-associated bleeding from BTC is suggested not only to be an effective and safe treatment option, but to improve the patient's quality of life. The further accumulation of cases will be required in order to confirm the usefulness of hemostatic RT for BTC.
\end{abstract}

Keywords: Biliary tract cancer; Bleeding; Hemostasis; Palliative radiotherapy; Quality of life

\section{Introduction}

Palliative radiotherapy (RT) for bleeding from gastrointestinal and genitourinary cancers is a useful treatment modality, able to control bleeding in 50-91\% [1-4]. Biliary tract cancer (BTC) can cause bleeding due to tumor invasion to the intestinal wall; however, it is often refractory because of the lack of definitive treatment modalities. While the survival time may be increased after RT [5-7], the usefulness of RT for managing tumor-associated bleeding (TAB) from BTC has not yet been investigated.

We herein report two patients who were endoscopically

Manuscript submitted April 14, 2018, accepted September 4, 2018

aDepartment of Gastroenterology, Kyoto Okamoto Memorial Hospital, Kyoto, Japan

bepartment of Surgery, Kyoto Okamoto Memorial Hospital, Kyoto, Japan 'Department of Radiology, Kyoto Okamoto Memorial Hospital, Kyoto, Japan ${ }^{d}$ Corresponding Author: Hideaki Kawabata, 58 Nishinokuchi, Sayama, Kumiyama-cho, Kuze-gun, Kyoto 613-0034, Japan.

Email: hkawabata@okamoto-hp.or.jp

doi: https://doi.org/10.14740/jmc3064e and histopathologically confirmed to have TAB from BTC and underwent hemostatic RT at our institution between 2016 and 2017.

\section{Case Reports}

\section{Case 1}

An 80-year-old man was pathologically diagnosed with lower extrahepatic cholangioadenocarcinoma in 2014. The tumor was localized, but an operation was not indicated because of cardiac dysfunction; therefore, only biliary drainage was performed. Two years after the diagnosis, he felt giddy and showed severe anemia, with a serum hemoglobin $(\mathrm{Hb})$ level of $4.4 \mathrm{~g} / \mathrm{dL}$. Computed tomography (CT) demonstrated a poorlyenhanced, low-density mass in the lower common bile duct, which invaded the duodenal wall (Fig. 1). Duodenoscopy revealed sustained oozing hemorrhage from the papilla due to invasive cholangiocarcinoma (Fig. 2). Palliative external-beam RT was attempted for the tumor of the papilla of Vater because his anemia was not improved after 16 units of blood had been transfused (Fig. 3). The radiation dose was 30 Gy in 12 fractions. After starting RT, neither progression of anemia nor treatment toxicity was observed. He received meals normally and spent 4 months at home until he died of hepatic failure due to multiple metastases.

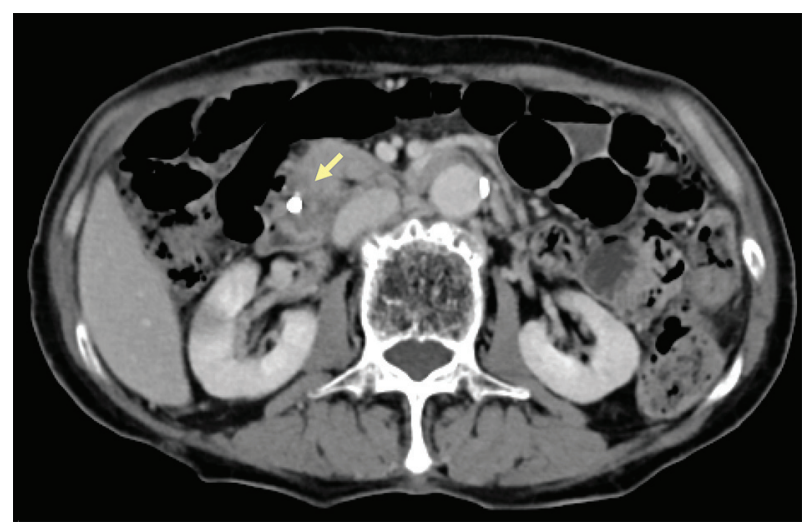

Figure 1. CT demonstrated a poorly-enhanced, low-density mass in the lower common bile duct, which invaded the duodenal wall (arrow). 


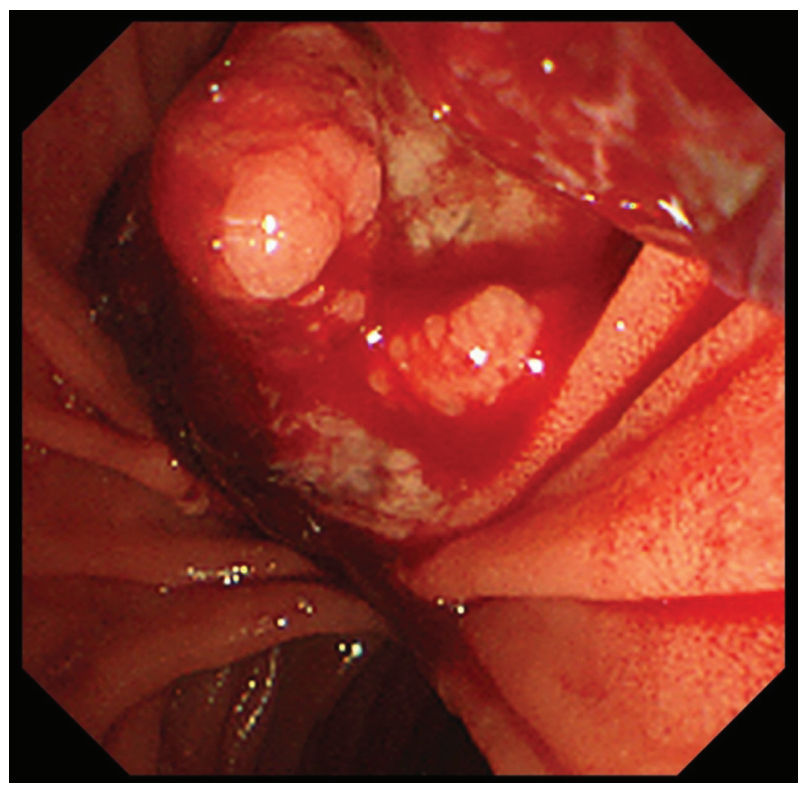

Figure 2. Duodenoscopy revealed sustained oozing hemorrhaging from the papilla of invasive cholangiocarcinoma.

\section{Case 2}

An 86-year-old man underwent choledochectomy and cholecystectomy with choledocho-jejunostomy for gallbladder adenocarcinoma in 2016. Ten months after the operation, he showed black stool and epigastralgia. The serum Hb level was $5.2 \mathrm{~g} / \mathrm{dL}$, and CT revealed both a poorly-enhanced, low-density recurrent mass at the porta hepatis, which invaded the duodenal wall, and an aneurismal dilation of the invaded-tumor vessel (Fig. 4). Duodenocsopy showed a red-colored, easily-hemorrhaged ulcer with submucosal growth appearance at the supraduodenal angle (Fig. 5), which was diagnosed histopathologically as adenocarcinoma. Palliative RT with 30 Gy in 10 fractions was in-

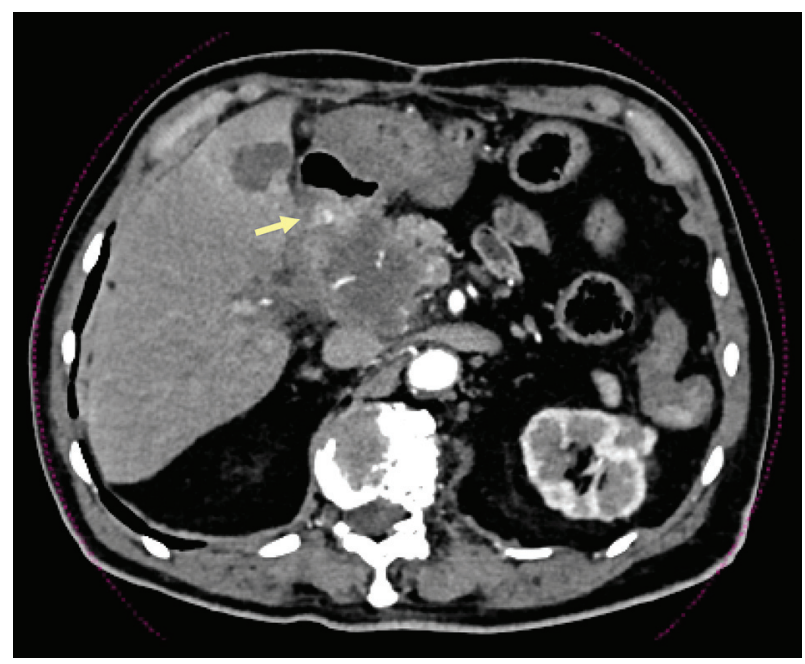

Figure 4. CT revealed both a poorly-enhanced, low-density recurrent mass at the porta hepatis, which invaded the duodenal wall, and an aneurismal dilation of the invaded-tumor vessel (arrow).

dicated for the recurrent tumor because conservative treatment including fasting, blood transfusion and the administration of a proton-pump inhibitor was not effective (Fig. 6). After starting RT, neither progression of anemia nor treatment toxicity was observed. He is still asymptomatic at 3 months after RT.

\section{Discussion}

The role of RT for BTC remains controversial due to the lack of large randomized trials. Therefore, RT is not recommended as a standard treatment, except for in a palliative setting, including for localized metastases or uncontrolled bleeding [8, 9]. Radiation gives rise to denudation of the surface of blood vessels, leading to thrombosis and capillary necrosis as the result of acute damage $[10,11]$. Favorable results of prolonging

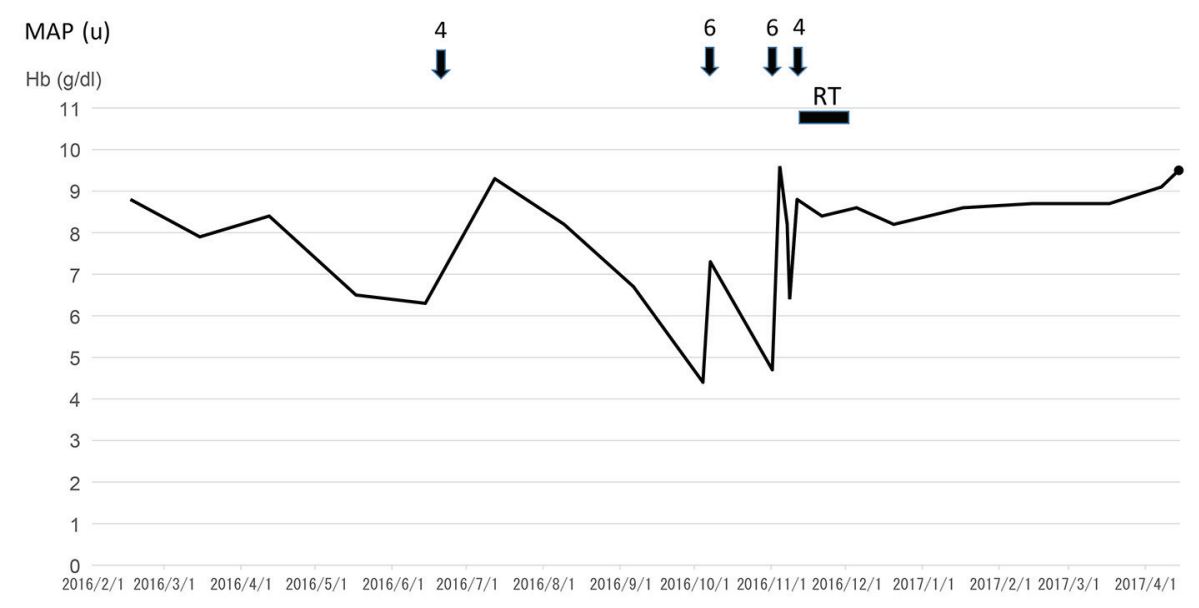

RT radiotherapy

Figure 3. The clinical course of case 1. 


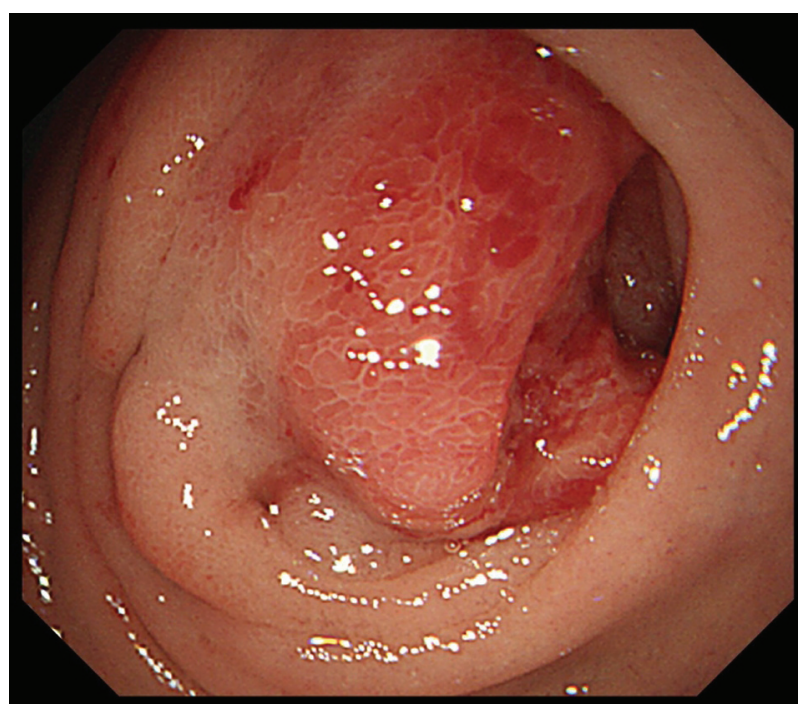

Figure 5. Duodenocsopy showed a red-colored, easily-hemorrhaged ulcer with submucosal growth appearance at the supraduodenal angle.

the survival time in BTC [5] and the reported efficacy for TAB in other cancers [12] suggest the hemostatic effect of RT in BTC; however, we found no reports describing the efficacy and safety of TAB from BTC in detail.

The effect of palliative RT on bleeding from gastric cancer has received more attention than that on bleeding from BTC. Small retrospective studies have shown a treatment success rate of $54-91 \%$ and a median duration of response ranging from 1.5 to 11.4 months with dose fractionation regimens of 6 - 60 Gy [1,2]. Grade 3 - 4 acute toxicities occurred in up to $15 \%$ of patients treated with RT alone [1]. Therefore, hemostatic RT for gastric cancer is suggested not only to be an effective and safe treatment option but to improve the patient's quality of life, although the optimum dose fractionation regimen is unclear.

In our cases, treatment success was achieved in both pa- tients, and they were able to spend their remaining life at home with no restriction on their oral intake. Furthermore, no adverse events were seen in any of the two patients after palliative RT. Therefore, palliative RT for TAB from BTC with 30 Gy in $10-12$ fractions is expected to be an effective and safe treatment modality.

\section{Conclusions}

Palliative RT for TAB from BTC is suggested not only to be an effective and safe treatment option, but to improve the patient's quality of life. The further accumulation of cases will be required in order to confirm the usefulness of hemostatic RT for BTC.

\section{Conflict of Interest}

No competing financial interests exist.

\section{References}

1. Tey J, Soon YY, Koh WY, Leong CN, Choo BA, Ho F, Vellayappan B, et al. Palliative radiotherapy for gastric cancer: a systematic review and meta-analysis. Oncotarget. 2017;8(15):25797-25805.

2. Kawabata H, Uno K, Yasuda K, Yamashita M. Experience of low-dose, short-course palliative radiotherapy for bleeding from unresectable gastric cancer. J Palliat Med. 2017;20(2):177-180.

3. Cameron MG, Kersten C, Guren MG, Fossa SD, Vistad I. Palliative pelvic radiotherapy of symptomatic incurable prostate cancer - a systematic review. Radiother Oncol. 2014;110(1):55-60.

4. Duchesne GM, Bolger JJ, Griffiths GO, Trevor Roberts J, Graham JD, Hoskin PJ, Fossa SD, et al. A randomized

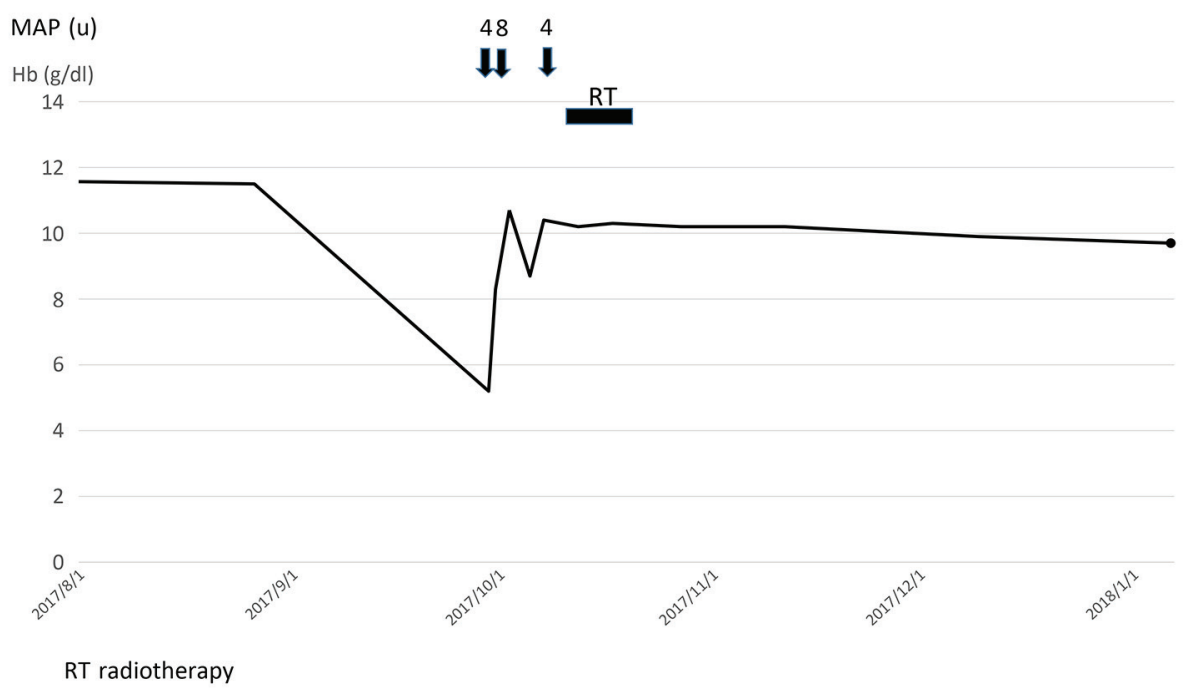

Figure 6. The clinical course of case 2. 
trial of hypofractionated schedules of palliative radiotherapy in the management of bladder carcinoma: results of medical research council trial BA09. Int J Radiat Oncol Biol Phys. 2000;47(2):379-388.

5. Macdonald OK, Crane $\mathrm{CH}$. Palliative and postoperative radiotherapy in biliary tract cancer. Surg Oncol Clin N Am. 2002;11(4):941-954.

6. Houry S, Barrier A, Huguier M. Irradiation therapy for gallbladder carcinoma: recent advances. J Hepatobiliary Pancreat Surg. 2001;8(6):518-524.

7. Shinohara ET, Mitra N, Guo M, Metz JM. Radiotherapy is associated with improved survival in adjuvant and palliative treatment of extrahepatic cholangiocarcinomas. Int J Radiat Oncol Biol Phys. 2009;74(4):1191-1198.

8. Khan SA, Davidson BR, Goldin RD, Heaton N, Karani J,
Pereira SP, Rosenberg WM, et al. Guidelines for the diagnosis and treatment of cholangiocarcinoma: an update. Gut. 2012;61(12):1657-1669.

9. Squadroni M, Tondulli L, Gatta G, Mosconi S, Beretta G, Labianca R. Cholangiocarcinoma. Crit Rev Oncol Hematol. $2017 ; 116: 11-31$.

10. Lee JA, Lim DH, Park W, Ahn YC, Huh SJ. Radiation therapy for gastric cancer bleeding. Tumori. 2009;95(6):726730.

11. Fajardo LF. The pathology of ionizing radiation as defined by morphologic patterns. Acta Oncol. 2005;44(1):13-22.

12. Cihoric N, Crowe S, Eychmuller S, Aebersold DM, Ghadjar P. Clinically significant bleeding in incurable cancer patients: effectiveness of hemostatic radiotherapy. Radiat Oncol. 2012;7:132. 